\title{
Itinéraires
}

Itinéraires Littérature, textes, cultures

2012-1| 2012

Genres et avant-gardes

\section{Quand le futurisme est femme : Barbara des couleurs}

Francesca Brezzi

\section{(2) OpenEdition}

Journals

\section{Édition électronique}

URL : http://journals.openedition.org/itineraires/1243

DOI : $10.4000 /$ itineraires. 1243

ISSN : 2427-920X

Éditeur

Pléiade

\section{Édition imprimée}

Date de publication : 1 septembre 2012

Pagination : 49-62

ISBN : 978-2-296-55776-5

ISSN : 2100-1340

Référence électronique

Francesca Brezzi, «Quand le futurisme est femme : Barbara des couleurs », Itinéraires [En ligne], 2012-1 | 2012, mis en ligne le 01 septembre 2012, consulté le 22 avril 2019. URL : http://

journals.openedition.org/itineraires/1243; DOI : 10.4000/itineraires.1243

\section{(ब) $(\Theta \Theta$}

Itinéraires est mis à disposition selon les termes de la licence Creative Commons Attribution - Pas d'Utilisation Commerciale - Pas de Modification 4.0 International. 


\title{
Quand le futurisme est femme : Barbara des couleurs ${ }^{1}$
}

\begin{abstract}
How can we explain the adhesion of many women to a pictorial current like Futurism, which was so misogynistic, aggressive and sometimes vulgar towards femininity? In this essay, first I aim to define the theoretic background of Futurism, i.e. the very ambivalent conceptual elaboration of the "female" which was expressed in those years and through that movement. Secondly, I consider a particular figure of woman and artist, Barbara, born as a Futurist, but ending up with completely different outcomes such as the peace movement and Feminism, in particular the empathic proximity to the texts of Luce Irigaray.
\end{abstract}

Keywords : futurism, feminine specificity, to start from oneself, art, philosophy Mots clés : futurisme, spécificité féminine, partir de soi, art, philosophie

Cet essai peut être présenté comme un palimpseste dont petit à petit on soulèverait les différentes couches. Le commencement est dans une question (sans exclure un mouvement de surprise) : comment expliquer l'adhésion de certaines femmes à un courant artistique aussi misogyne, agressif, parfois même vulgaire à l'égard de la féminité, que le futurisme? Et de fait, la présence de femmes, connues et appréciées à leur époque, a été quantitativement et qualitativement importante au sein du mouvement. De ce constat - première couche du palimpseste - dérivent des questionnements de type historiographique : repenser la place des femmes dans les mouvements d'avant-garde, mais également l'articulation entre genre (gender) et genre (littéraire)? Ces questionnements sont le résultat de l'effort accompli par la réflexion féministe pour redécouvrir le mouvement

1. Ce titre évoque celui de notre livre Quand le futurisme est femme. Barbara des couleurs, trad. de l'italien Bernadette Rigaud, Paris, Mimesis/France, 2010, auquel nous renvoyons pour une réflexion plus élaborée. Toutes les traductions de cet article sont de Bernadette Rigaud. 
futuriste. Mais, si d'un côté, nous ressentons avec force l'exigence de porter à la lumière une réalité souterraine (dans ce groupe comme dans d'autres), de nous opposer à l'effacement de la présence historique des femmes, de focaliser notre attention sur les points importants que sont la relation entre les femmes et l'avant-garde, les femmes et la création artistique, etc., d'un autre côté - deuxième couche du palimpseste - s'impose aussi la réflexion théorique sur ce que nous pourrions préalablement définir comme la conception idéologique du futurisme, à savoir l'élaboration conceptuelle sur le "féminin 》 (la femme et la question féminine), dans les romans, les essais-manifestes des femmes, textes d'où découla par la suite la mise en pratique effective de cette élaboration.

Les réponses à ces questionnements ne sont ni simples ni univoques, mais il faut les rechercher en creusant dans ce monde complexe mais aussi prismatique qu'a été le futurisme.

Si l'on s'arrête sur quelques textes de Marinetti et si on se met à l'écoute de la voix personnelle des femmes, on aperçoit les contradictions internes du futurisme. Il y a tout d'abord le fameux « mépris de la femme ${ }^{2}$ », répété à maintes reprises et soutenu idéologiquement par Marinetti et d'autres représentants du futurisme qui identifient la femme avec le sentiment et se battent donc contre l'amour romantique et le mariage et pour l'exaltation de l'amour libre. Il en découle des corollaires décisifs comme la théorisation de l'infériorité cérébrale de la femme, ainsi que de sa férocité ; tout comme se manifeste avec fréquence un « virilisme exhibitionniste et maniaque ${ }^{3}$ » au ton et au contenu belliqueux et nationalistes. Mais tout cela constitue en fait un amalgame très équivoque, puisque l'auteur envisage parfois une révolution dans la sphère privée tout en maintenant inaltérés les termes de la condition féminine, reconnaît l'origine historique de l'infériorité de la femme tout en manipulant cette inégalité, ou encore attaque l'institution du mariage et simultanément codifie le rôle maternel. Par ailleurs, cette ambiguïté s'accompagne d'un manque d'innovation. Le propos prend en effet un ton conservateur et fait apparaître clairement ses liens étroits avec les théories de la fin du XIX ${ }^{\mathrm{e}}$ siècle, comme le positivisme matérialiste de l'école de Lombroso, mais aussi l'influence des penseurs allemands comme Nietzsche, Schopenhauer, Moebius et Weineger.

L'autre moitié du futurisme, défini justement comme le plus misogyne des courants de l'avant-garde, est constituée par les nombreuses femmes, écrivaines, poétesses, artistes, musiciennes, danseuses ou polémistes,

2. Filippo Tommaso Marinetti, Mafàrka le füturiste : roman africain, Paris, Sansot, 1910, p. VIII.

3. Rita Guerricchio, « Donne nell'avanguardia », La Donna nella letteratura italiana del Novecento, Empoli, mai 1981, numéro unique, p. 32. 
souvent reléguées au statut de simples disciples ${ }^{4}$, mais qui n'en participent pas moins au mouvement et dont les attitudes criardes apparaissent transgressives par rapport à la morale commune; pour elles, l'adhésion à une esthétique révolutionnaire permet en effet de mettre en cause les rôles traditionnellement attribués aux femmes.

Avant de rappeler l'auto-caractérisation des nombreuses artistes qui ont participé au futurisme, il faut introduire un questionnement de type plus sociologique: les avant-gardes, en tant que mouvements collectifs, ont cherché à articuler, en termes de révolution ou de rupture, une transformation radicale de la vie sociale, politique et intime et une transformation tout aussi radicale des pratiques artistiques et de la pensée de l'art. En Italie, les femmes tentées par l'aventure futuriste sont nombreuses, « toutes dotées quand même d'un surplus d'audace pour choisir d'appartenir à un mouvement caractérisé par un taux élevé de provocation ${ }^{5} \gg$. Surplus de rupture : sortie de l'espace de la chambre traditionnellement réservé aux femmes, possibilité de provoquer des interférences éclatantes dans la sphère publique. C'est là une première piste à suivre pour répondre à nos questions : l'adhésion au futurisme représente pour nombre d'artistes un défi et un acte concerté de destruction et de démantèlement, un défi à l'esprit d'abnégation et de sacrifice considéré jusqu'à cette époque (et même par la suite) comme la finalité de la vie des femmes, une destruction des stéréotypes féminins, qui fait s'interpénétrer, dans la plus grande exaltation et la plus grande exubérance, la sphère esthétique et la vie, où la fantaisie et la fonction de l'imaginaire deviennent la clé susceptible d'ouvrir de nouveaux mondes.

Cette recherche d'une attitude insolite et d'une identité inédite se heurte à un contexte historique peu favorable. Les principes théoriques, les découvertes et les innovations du futurisme ne sont en effet pas élaborés par les femmes futuristes, mais acceptés de manière passive, ce qui détermine par la suite des ambiguïtés, ou une bifurcation possible autour de la question féminine, aussi bien chez les hommes que chez les femmes : d'un côté, le geste transgressif et anti-passéiste, les écritures profondément polémiques,

4. Les femmes donnent même le jour à de nouveaux langages, à des formes artistiques inhabituelles, tout au moins pour l'époque, dans les domaines les plus communs, comme la peinture et la littérature, mais aussi sur des terrains nouveaux et mixtes comme l'art tactile, la danse, etc. Parmi cette inventivité stylistique, on peut citer, par exemple, les "parolibere » (tables de mots libres), qui ne sont pas simplement l'expression de la cohabitation de mots et d'images, mais de véritables mots iconiques. Compagne et femme de Marinetti, Benedetta Cappa est peintre et écrivaine et elle compose des tableaux tactiles. Il faudrait aussi signaler les artistes qui donnent vie à la danse "antipsychologique ", les auteures du manifeste de "l'aéroplastique futuriste » (Regina et Barbara, comme nous verrons) ou encore l'intérêt pour la mode (Alma Finora).

5. Claudia Salaris, "Incontro con le futuriste ", dans Laura Iamurri et Sabrina Spinazzè (dir.), L'arte delle donne nell'Italia del Novecento, Rome, Meltemi, 2001, p. 50. 
sans parler des éclats verbaux et de l'adhésion aux nouveaux mythes de la machine, de la vitesse, de la métropole; de l'autre, une pratique futuriste et des prises de position se manifestant par des affirmations de caractère complètement opposé, c'est-à-dire conciliantes et serviles vis-à-vis de l'idéologie machiste. Et cela vaut pour les représentations de la masculinité, comme pour celles de la féminité ou de tout déplacement de ce binarisme. En posant un regard diachronique sur ces deux attitudes, on peut presque percevoir un premier et un second futurisme, surtout si l'on n'oublie pas que la production féminine appartient presque entièrement à la période finale du futurisme, à savoir aux années où une dimension idéologique restauratrice freine la poussée anticonformiste initiale pour aboutir à la conversion fasciste.

Quelle est donc la voix des femmes? Pour répondre, il faut se tourner vers l'auto-caractérisation des nombreuses artistes qui participent au futurisme, en d'autres termes, focaliser son attention sur la manière dont elles abordent elles-mêmes le thème de la femme, sans perdre de vue - à l'arrière-plan - que ces mêmes années du $\mathrm{xx}^{\mathrm{e}}$ siècle voient s'affirmer les premières formes du féminisme et les premiers pas du long chemin qui conduit à l'émancipation des femmes. Concernant l'Italie, on peut penser entre autres à Anna Maria Mozzoni, à Anna Kuliscioff et à ce « Féminisme » que Valentine de Saint-Point définit comme « une erreur politique [...] une erreur cérébrale de la femme $»^{6}$.

La voix des femmes se fait ainsi entendre chez Valentine de Saint-Point elle-même, première femme à adhérer au courant futuriste et qui a rédigé un « Manifeste de la femme futuriste » (1912) et un « Manifeste futuriste de la Luxure » (1913). Dans le premier, elle reproduit avec force les positions de Marinetti, affichant son mépris pour une humanité médiocre, radicalisant la distinction entre sexe et sentiment et exaltant tout d'abord des figures féminines comme les Érinyes ou les Amazones, puis la figure de l'androgyne. Contre l'instinct maternel, au nom d'un refus de toute forme de convention et pour l'indépendance des femmes, Valentine de Saint-Point, dans son « Manifeste futuriste de la Luxure », expose à grands traits une « joie de la possession et de la domination ${ }^{7}$ » et elle invoque une cruauté masculine valable pour les deux sexes, le but ultime résidant précisément dans la négation de la différence et le refus de la fonction génératrice de la féminité. Il y a là un thème que le deuxième aspect du futurisme, défini d'abord comme mouvement de proposition, laissera plus tard tomber au nom d'un « futurisme de l'espèce ${ }^{8}$ » qui exaltera le rôle traditionnel de

6. Valentine de Saint-Point, "Manifeste de la femme futuriste» [1912], repris dans Manifeste de la femme futuriste, Paris, Mille et une nuits, 2005, p. 12.

7. Valentine de Saint-Point, "Manifeste futuriste de la Luxure» [1913], repris dans Manifeste de la femme futuriste, op. cit., p. 22.

8. Italo Tavolato, Contro la morale sessuale [1913], cité dans Mario Verdone, Prosa e critica futurista, Milan, Feltrinelli, 1973, p. 240. 
la femme, dissertant sur la femme souche, la femme race, confirmant les conceptions anthropologiques positivistes, régressant même à une vision de la féminité comme fonction biologique. C'est dans ce cadre que va éclore une riche production, très souvent masculine, qui par la suite s'imbriquera étroitement avec les thèses du fascisme sur la maternité comme devoir envers l'État, sur l'exaltation de la mère exemplaire, etc.

Attitude insolite et recherche d'une identité inédite : ces deux caractéristiques se retrouvent exemplairement chez la figure intéressante bien qu'elle-même ambigüe de Rosa Rosà. Son nom véritable est Edith von Haynau. Autrichienne issue d'une famille noble mais en rupture avec les règles de son milieu, elle s'installe en Italie pour se consacrer aux arts. Elle est peintre (mots libres), femme de lettres, auteure d'un roman intitulé Una donna con tre anime (1918), illustratrice de textes dans la revue Italia futurista et elle signe de façon significative en 1916 le manifeste « La science futuriste » où « cérébralité et animisme s'imbriquent pour composer une nouvelle vision de la science, en mesure d'élargir ses propres horizons dans la mesure où ils s'associent aux démarches artistiques ${ }^{9}$ ».

Dans ce contexte, Rosà se distingue pour plusieurs raisons : en premier lieu, elle apparaît comme « engagée, tout au moins d'un point de vue théorique, à dépasser les limites de la misogynie futuriste, en vue d'esquisser une perspective de proto-féminisme ${ }^{10} »$. En deuxième lieu, elle s'inscrit dans un contexte bien particulier qui voit s'accroître le rôle de la femme pendant et après la guerre, et elle souligne que personne ne songe à mettre en lumière l'importance de cette nouvelle activité féminine, ce qui la conduit à réfléchir sur les changements qui en découlent, non seulement dans la vie publique, mais aussi dans l'espace privé. Elle manifeste la volonté de dessiner la « femme de l'après-demain », dotée d'un « moi libre immortel, qui ne se donne à personne ni à rien », une femme qui veut atteindre un «métacentre abstrait, impossible à conquérir, inaccessible aux séductions les plus expertes, inaccessible aux consommateurs de remontants type Fernet $\gg^{11}$. Par ailleurs, cette figure féminine se doit de surmonter le rôle maternel traditionnel, qui ne permet pas d'atteindre le moi conscient, autonome et indépendant. Mais, surtout et en même temps, Rosà

9. Rita Guerricchio, « Donne nell'avanguardia », op. cit., p. 44. À ce propos on peut rappeler le débat, expression du deuxième filon du futurisme déjà mentionné, apparu au grand jour en pleine guerre mondiale et qui a animé pendant un an L'Italia futurista. À côté des pages politiques de Marinetti, de son bellicisme sans retenue («marcher et ne pas pourrir» était sa devise, adoptée par la revue) se développe la tendance magique-occultiste, ésotérique, onirique, hérétique par rapport au futurisme classique mais dans laquelle la présence des femmes est importante. Rappelons, entre autres, Maria Ginanni, Fulvia Giuliani, Irma Valeria, Eva Khun Amendola et Rosa Rosà.

10. Anna Nozzoli, Tabù e coscienza, Firenze, La Nuova Italia, 1978, p. 53.

11. Rosa Rosà, « Le donne cambiano finalmente ", L'Italia futurista, n 27, 26 août 1917, p. 2. 
s'interroge avec inquiétude sur l'identité féminine : « femme-objet, femme négligeable, femme illogique inconsistante, irresponsable, femme stupidité, femme poupée [...]. Constat consécutif à son infériorité méprisable. Ou bien femme indépendante, affranchie, laide, intelligente, caustique, désagréable car non désireuse de plaire ${ }^{12} \gg$ ? Et elle termine avec mélancolie par cette question, « qui peut me dire comment il faut être ${ }^{13}$ ? », dans laquelle l'importance de ce que l'on peut nommer le « partir de soi ${ }^{14} »$ est méconnue, alors qu'elle est ailleurs presque entrevue et ressentie.

On peut conclure que dans l'ensemble les écrivaines, même si elles sont incapables d'écarter les stéréotypes de l'époque qui leur sont préjudiciables, prônent un modèle de femme non féministe mais quand même émancipée ${ }^{15}$. Elles expriment toutefois aussi de lourdes ambiguïtés, comme l'individualisme exaspéré qui tend au mythe de la femme supérieure théorisé par Valentine de Saint-Point ou par Fulvia Giuliani : « Je me sens futuriste. Je suis devenue telle presque sans le savoir [...] je ne suis pas une machine incubatrice... que vous appelez femme. J'appartiens aux ÉLUES ${ }^{16}$. » L'aboutissement, presque naturel, de ces théorisations sera l'imbrication avec le fascisme féminin et donc avec les mythes de la nation et des anciens combattants. L'élaboration théorique du futurisme subit ainsi une véritable involution, en particulier pour ce qui est de certaines thématiques comme la maternité. Anna Gozzoli voit dans le lien entre futurisme et fascisme le point d'arrivée d'une trajectoire hétéroclite et ambiguë à l'image de la trajectoire plus spécifique de l'idéologie futuriste sur la femme (une trajectoire commencée «par les lueurs incandescentes de la polémique de Marinetti et conclue par la restauration moralisatrice du régime ${ }^{17} \gg$ ) dont l'élément de cohésion a toujours été la catégorie essentielle du virilisme.

Peut-être pourrions-nous essayer d'avancer une réponse à nos questionnements initiaux, en définissant l'attitude de nombre de femmes

\section{Ibid.}

13. Ibid. Ces éléments contradictoires et énigmatiques se rencontrent concrètement dans les romans de Rosà, romans que nous ne pouvons pas examiner, mais dans lesquels la polémique anti-machiste et la lutte pour reconquérir l'identité féminine s'estompent et se corrompent souvent pour aboutir à des solutions ambiguës, voire opposées.

14. Le « partir-de-soi » est une catégorie propre au féminisme italien qui renvoie à l'expérience vécue. Valable pour les hommes comme pour les femmes, il ne constitue pas un abandon complaisant à sa propre intériorité, mais il décrit une modification de soi à partir d'un vide du sens et dans le passage d'un point d'appui à un autre: les images et les émotions sont les pierres invisibles avec lesquelles notre maison intérieure se construit à travers les ans.

15. Voir Elisabetta Mondello, La rivoluzione apparente di F.T. Marinetti, Roma, Editori Riuniti, 1987, p. 37.

16. Fulvia Giuliani, «Continua la polemica femminile con Vianello », Roma futurista, $2^{\mathrm{e}}$ année, $\mathrm{n}^{\circ} 13,30$ mars 1919 , p. 3.

17. Anna Nozzoli, Tabù e coscienza, op. cit., p. 63. 
futuristes comme celle d'une deuxième naissance. En effet, ce n'est pas un hasard si presque toutes adoptent un pseudonyme, comme si elles étaient à la recherche d'une nouvelle identité : dans l'Italie provinciale et arriérée de l'époque, le défi est celui de prendre en main sa propre existence, dans l'illusion d'être reconnue et de trouver des espaces d'autonomie au sein d'un mouvement provocateur qui, même dans sa grande nébulosité et même s'il s'agit uniquement d'éclairs hétérogènes, présents surtout dans l'expérimentation et bien vite dispersés après vingt ans de fascisme, sait parfois appréhender la position subalterne féminine comme un produit de l'histoire et de la morale, et non comme le fruit de déterminations biologiques.

Ce tour d'horizon consacré à l'autre moitié du futurisme nous permet de mieux appréhender le parcours particulier de Barbara. Si l'ensemble de ses œuvres artistiques constitue un voyage dans son intériorité personnelle, il s'agit de comprendre les motivations théoriques qui la guident dans sa trajectoire, en suivant la réflexion rétrospective qu'elle livre dans son autobiographie de plus de cinq cents pages qui réunit des faits et des moments heureux ou dramatiques, des sentiments et des émotions, mais qui est surtout marquée par des couleurs ineffables, des éclats de lumière et de flammes, qui permettent de mettre en évidence son identité telle qu'elle la raconte et la construit.

À travers le cas de Barbara apparaît l'esquisse de ce "partir de soi » qu'on ne retrouve pas chez les futuristes préalablement examinés. D'abord futuriste, elle trouve par la suite d'autres points d'ancrage très différents, comme le pacifisme et le féminisme, notamment à travers son affinité empathique avec les textes de Luce Irigaray. Sa vie présente un caractère prismatique. S'y entremêlent l'art et la vie, un binôme qui dans le futurisme apparaissait comme une forme de provocation, alors que, en revanche, des mots et des œuvres de Barbara transparaît le chiffre de son identité de femme, conquis seulement après avoir parcouru un labyrinthe, le labyrinthe de Barbara.

Femme née d'elle-même, déclare à maintes reprises Barbara, en revivant ses nombreuses vies rappelées à présent brièvement. Elle naît dans une petite ville italienne en 1915 et son vrai nom est Olga Biglieri. Elle grandit dans un milieu bourgeois et conservateur et elle manifeste rapidement une tendance à la rébellion, à l'anticonformisme qui la pousse vers des voies alternatives : rejet du modèle féminin traditionnel et recherche de relations égalitaires avec les hommes, aussi bien dans les sports que dans les arts. Deux passions s'imbriquent chez cette jeune femme : la peinture et le vol. Faisant la preuve de son caractère aventureux et anticonformiste, elle est ainsi la première et l'unique femme à fréquenter une école de vol à voile et elle obtient le brevet de pilote à seulement dix-huit ans. Son premier tableau, Vomito dall'aereo (1938), s'inspire des sensations éprouvées en vol et il révèle donc un monde insolite fait de contrastes chromatiques, de perspectives déformées et tournantes, d'où se dégagent les lignes de force 
du mouvement et de la vitesse. Elle dit elle-même que le point de départ de sa peinture est dans une action déjà « déformante ${ }^{18}$ » et cela avant même sa rencontre avec le futurisme.

Après être entrée en contact avec le groupe futuriste de Vérone, elle adopte le pseudonyme de Barbara comme pour se donner « une nouvelle naissance ${ }^{19} 》$, qui évoque selon elle quelque chose de primordial, de barbare et de fort à la fois. À la Biennale de Venise de la même année 1938, elle expose un tableau de grande dimension, L'Aeroporto abbranca l'aeroplano, signé : Barbara aviatrice futuriste. Le défi est lancé : femme et jeune, elle devient un cas et ne passe pas inaperçue dans le mouvement futuriste, car elle possède une conscience d'elle-même peu commune. L'adhésion au futurisme marque ainsi une riche période de création artistique caractérisée par la production d'œuvres importantes, exposées à la Quadriennale de Rome avec celles de cinq autres femmes seulement, et petit à petit dans d'autres manifestations organisées par les futuristes, comme l'Exposition d'outre-mer à Naples et la XXII ${ }^{\mathrm{e}}$ Biennale. Barbara - que Marinetti définit comme une « femme peintre aérienne géniale ${ }^{20} »-$ expose des peintures à l'huile: Pensieri in carlinga, Festa di porto mediterranea et Areopittura di città, jugées par Giorgio di Genova « bien plus mûres que les précédentes, aussi bien pour la mise en page de la vision subjective des agglomérats urbains sous-jacents saisis par la carlingue, que pour le caractère dynamique des scènes ${ }^{21} »$. Ces peintures, revisitées bien des années plus tard, expriment - au dire de l'auteure elle-même - le rapport étroit entre l'art, la vie et l'histoire et, sous des formes différentes, elles continuent à représenter son cheminement. Dans l'avant-garde futuriste, rappelle encore Barbara, si on voulait « tuer le clair de lune, on voulait aussi transformer l'Italie en une œuvre d'art ${ }^{22} »$.

Cette période exaltante prend brusquement fin avec le déclenchement de la Seconde Guerre mondiale, à laquelle son mari, le poète et écrivain futuriste Ignazio Scurto, participe et dont il ressort éprouvé physiquement et moralement. Touchée dans sa vie personnelle, Barbara envisage à présent différemment le bellicisme du futurisme. Loin de juger la guerre

18. Olga Biglieri Scurto, Barbara dei colori, Roma, Centro Internazionale Antinoo per l'Arte, 1998, p. 203.

19. Ibid., p. 220.

20. Ibid., p. 267.

21. Giorgio di Genova, Barbara dal futurismo al 2001, catalogue publié à l'occasion de l'exposition qui s'est tenue à Rome en avril 2001, Roma, Centro Internazionale Antinoo per 1'Arte, 2001, p. 24. Les autres femmes peintres étaient Leandra Angelucci, Regina, Magda Korompay et Benedetta. Ces tableaux ont aussi été présentés dans une exposition qui s'est tenue en mars 1983 à la galerie L'Alzaia de Rome et qui a donné lieu à la publication d'un petit catalogue, Barbara Areopittrice Aviatrice futurista, par Claudia Salaris, Edizioni Alzaia (s. d.).

22. Voir Olga Biglieri Scurto, Barbara dei colori, op. cit., p. 294. 
comme « l'hygiène du monde », elle se pose des questions qui lui semblent urgentes : «Qui avait inventé ce chaos de la guerre? [...] Qui commandait, qui avait le pouvoir? Pour quelle raison les futuristes, qui avaient à leur tête Marinetti, exaltaient-ils donc ce désastre ${ }^{23}$ ? » Le dernier tableau de cette époque, Battaglia aerea, reflète ses inquiétudes, ainsi que son détachement intime du credo de Marinetti : deux avions se battent en duel dans les cieux, un autre tombe en laissant derrière lui une traînée obscure, tandis qu'un profil de femme regarde la scène d'en bas :

l'avion, ici, n'est plus le symbole de la force et de la joie de voler : ces valeurs et ces sensations pour lesquelles je suis devenue futuriste. C'est une machine que l'homme jette vers la destruction et vers la mort. Non, je ne peux pas exalter la guerre. Je veux décider de moi-même, envisager la vie comme désir; je n'accepte pas le fait que d'autres se soient arrogés le droit de programmer pour moi cette chose que je ne comprends pas et qui me semble absurde et inhumaine [...] je n'étais pas d'accord; pour moi, privée de mes droits d'épouse et de mère, la guerre était un fait dramatique et destructeur, se résumant en un seul mot, tuer ${ }^{24}$.

Dans un moment d'une telle gravité, ces mots prononcés par une Barbara qui souffre de certaines blessures jamais cicatrisées unissent avec sincérité et de manière prégnante son vécu existentiel et sa propre conscience d'artiste, et nous pouvons y lire les racines de son engagement pour la paix dans les années 1970 et de son adhésion au mouvement pacifiste qui la mettra en contact avec le drame du Japon ${ }^{25}$.

Dans les années suivantes, elle conjugue encore de manière étroite son Erlebenis de femme - la participation au mouvement féministe au sein de la Gauche - et son expérience dans le monde des arts.

Si la place manque ici pour suivre la trame complexe de la vie de Barbara, qui meurt en janvier 2002, nous voudrions toutefois souligner de quelle manière le thème de l'imbrication entre l'art et la vie revient encore, peut-être de manière plus consciente, dans la seconde partie de son existence, comme harmonie et enrichissement mutuel d'une recherche individuelle et d'un engagement féministe, intense et absorbant. En témoignent

23. Ibid., p. 91.

24. Ibid., p. 92.

25. Son art devient un moyen de communication solidaire et de partage, une arme pacifique pour les luttes, mais aussi le chiffre d'une créativité qui se met au service d'une cause grande et juste. Le projet de l'Arbre de la paix est emblématique des années 1980 et de cet engagement : un rouleau de papier à détrempe de dix mètres de longueur, parsemé de nombreuses empreintes de mains trempées dans la couleur, un arbre long et délicat aux racines pas tout à fait ensevelies, un arbre comme symbole de la vie qui grandit dans l'harmonie de l'homme avec lui-même, avec les autres hommes, avec la nature. En 1986, à l'occasion du quarante-et-unième anniversaire du lancement des bombes atomiques, l'Arbre est offert par Barbara au Memorial Museum de Hiroshima, lors d'un voyage passionnant et particulièrement émouvant. 
exemplairement les noyaux centraux de l'activité picturale de cette artiste née avec le futurisme et arrivée bien loin de lui, à savoir la période placentaire suivie par la création noétique, noyaux qui sont soutenus par une élaboration théorique de type philosophique sous le signe de la femme.

Une série de tableaux, définis par Barbara comme placentaires, représentent, en un certain sens, le moment propédeutique à la période noétique, qui mûrit tout de suite après. Les premiers tableaux, remontant aux années 1979-1981, sont créés significativement après la mort de sa mère et au moment où en Italie le thème de l'avortement fait l'objet de discussion (1981). Si, sur le plan rationnel, ces œuvres expriment un cheminement associé à l'être femme, et donc à la différence sexuelle, « qui raconte le fait d'engendrer, au plan symbolique ils [sont] indissolublement liés [...] aux liens intimes, mystérieux, poignants qui étreignent une fille à sa mère ${ }^{26}$ "».

Se trouvant occupée à démeubler et à vider la vieille maison maternelle, lieu de mémoires, Barbara, presque par hasard, trace quelques signes colorés en utilisant de vieilles détrempes de ses filles : « et ce fut à partir de ces couleurs que ma main et mon pinceau donnèrent sa forme au premier placenta : visualisant la grande promesse et en même temps le drame de la féminité qui engendre une vie en sachant qu'elle est destinée à mourir ${ }^{27} \gg$. À l'occasion de la Fête des femmes de Venise, en juillet 1981, Barbara n'expose plus de « vieilles choses futuristes » mais le fruit de sa recherche la plus récente : « avec ces deux placentas j’exposais vraiment mes entrailles, le niveau le plus intime et douloureux de moi-même, de ma vie [...], chaque placenta était "enceinte" : accueillait en son sein les germes d'une vie nouvelle qui représentaient comme des corpuscules élémentaires, frangés, presque des fleurs $\gg{ }^{28}$. À partir de ce moment, elle parle avec plus d'insistance d'une peinture de femme qu'il faudrait faire émerger. C'est ainsi qu'après des années de silence Barbara revient à la peinture. Partant d'elle-même, elle caractérise ainsi son objectif : « Je voulais m'exprimer, tirer hors de moi des sensations que les mots ne pouvaient contenir. Je remplaçais les mots par le cœur ${ }^{29}$. $\gg$ Les tableaux placentaires cherchent à exprimer la double racine - biologique et historique - de la spécificité féminine, mais aussi à témoigner de ce que la production artistique renferme l'imaginaire, l'émotion, le ressenti personnel, imbriqués avec la recherche conceptuelle, comme nouvelle relation entre la subjectivité, l'idée et la réalité. «Le corps magique de la femme est exploré de l'intérieur, dans sa partie la plus secrète et la plus précieuse ${ }^{30}$. »

26. Olga Biglieri Scurto, Barbara dei colori, op. cit., p. 314.

27. Ibid., p. 312.

28. Ibid., p. 357.

29. Giorgio di Genova, Barbara dal futurismo al 2001, op. cit., p. 15.

30. Olga Biglieri Scurto, Barbara dei colori, op. cit., p. 328. 
Quel chemin parcouru depuis les considérations futuristes sombres et fanatiques sur la maternité ! L'adhésion au futurisme lui apparaît à présent comme un sentier inachevé et nécessairement interrompu.

La pensée de l'artiste sur la spécificité féminine dans l'art et dans la culture atteint alors son sommet, sans se détacher cependant du concret, cet agir effréné qui lui permet d'emprunter de nouveaux sentiers, dont elle reconnaît la richesse liée au fait de se retrouver, de communiquer et de chercher continuellement entre femmes. Tout cela aboutit à sa relation avec une philosophe influente, Luce Irigaray, mais cette rencontre idéale est comme anticipée dans cette phase créatrice lors de laquelle, de manière plus ou moins consciente, Barbara vit en son sein les résultats de la réflexion féministe et les rend visibles dans sa peinture. Dans Je, tu, nous : pour une culture de la différence, Irigaray décrit une « relation placentaire ${ }^{31}$ » dans laquelle elle aborde l'heureuse complexité relative à la thématique du corps : d'un côté, l'empreinte physique essentielle, la relation avec les processus matériels de la biologie et, de l'autre, l'élément symbolique, culturel et spirituel, une complexité que le féminisme a été le premier à mettre en évidence, réfléchissant sur les grandes questions implicites dans l'ambiguïté du corps et de la vie - naissance, mort, maladies - dérivant de ces deux racines. Corps et vie sont ainsi à la fois liés aux processus matériels de la biologie et inscrits dans les développements de la culture. Irigaray dessine l'être de la femme comme potentialité du duel, reconnaissance ou passion de l'autre, vie qui devient ainsi chair vive et spirituelle et, donc, relationalité impossible à éliminer. Les mots de Barbara retentissent encore : « le placentaire exalte la clef de voûte du corps de la femme, [...] l'enveloppe de la créativité biologique. Voir avec fantaisie pour découvrir le divin, l'illustrer avec des couleurs et marquer avec l'empreinte d'une fleur la vie nouvelle ${ }^{32} »$.

Cette élaboration aboutit à la peinture noétique, que Barbara développe là encore en étroite amitié avec d'autres femmes et dont elle retrouve la suggestion exemplaire dans la philosophie de Luce Irigaray.

Ce mouvement, important et singulier dès sa genèse, prend naissance à partir des échanges, des réunions, des discussions et des dialogues entre femmes artistes. Toutefois, il s'agit là d'un nouveau cheminement, d'un voyage peut-être, voire d'une lutte à engager contre des résistances consolidées. Libres de toutes appartenances idéologiques, corsaires ou indisciplinées, ces femmes saisissent l'importance de l'expérimentation par laquelle elles veulent presque elles aussi - comme les femmes futuristes - se donner une nouvelle naissance.

31. Luce Irigaray, Je, tu, nous : pour une culture de la différence, Paris, Grasset, 1990, p. 46.

32. «Barbara parla di Barbara », Barbara dal futurismo al 2001, op. cit., p. 76. 
Si telles sont les motivations de Barbara, le contenu de son travail réside dans l'élaboration d'une peinture de femme, que le terme noétique résume de manière intentionnellement hermétique : «le chemin de la femme vers l'art n'est pas un chemin technique, rationaliste, il ne naît pas d'une pensée abstraite et bien organisée [...]; la femme réunit l'esprit et le corps, l'art et la vie, la pensée et l'action. Elle procède par connaissance intuitive, par noèse précisément ${ }^{33} \gg$.

La rencontre avec un texte de Luce Irigaray, Passions élémentaires, est fondamentale pour Barbara :

la mienne ne fut pas une simple lecture du livre : je suis entrée mystérieusement en syntonie avec Luce, avec son expérience, sa pensée [...] à un certain point, une véritable unité féconde s'est créée entre ses mots-pensée-vie et ma peinture-pensée-vie. Et ainsi ses mots sont entrés dans mes tableaux : ils devinrent eux-mêmes signes et couleurs ${ }^{34}$.

Une syntonie de fond, issue de la rencontre de deux sensibilités féminines, dans l'union d'une recherche picturale et de l'effort spéculatif : telle est l'émotion ressentie par l'artiste.

L'empathie entre les deux femmes réside notamment dans la caractérisation du sujet féminin comme une identité constituée non seulement de logos mais de catégories originaires, soit des passions comme l'admiration, le désir, la caresse, qui représentent les étapes fondamentales de ce voyage identitaire. Contre le platonisme de notre tradition métaphysique, il s'agit de récupérer la sève vitale qui circule dans les racines de l'homme et de la femme (récupérer ce que les premiers philosophes et les anciens sages avaient compris et accepté avec naturel) et de constater qu'il est impossible de scinder le corps et la raison, scission qui constitue bien au contraire une menace pour la pensée. Il faut remettre les passions à leur juste place, entre l'homme et la femme. Barbara fait écho aux paroles d'Irigaray quand elle souligne la manière dont la contribution des femmes à la vie artistique constitue « un point névralgique pour le développement de l'humanité : la reproduction biologique et ses conditions quotidiennes, la sexualité et l'affectivité, le rapport entre personnel et politique $\left.{ }^{35}\right\rangle$. Le but ultime, pour Irigaray, est ainsi la constitution d'une éthique qui, posant comme pierre angulaire les passions toujours réprimées, non dites, représente une éthique nouvelle, l'éthique de la différence sexuelle que la philosophe esquisse par des métaphores audacieuses, suggestives et somptueuses.

33. Olga Biglieri Scurto, Barbara dei colori, op. cit., p. 360.

34. Ibid., p. 370. Dans Quand le futurisme est femme, nous proposons une lecture parallèle de plusieurs textes d'Irigaray associés à la peinture de Barbara, pour saisir des correspondances ou, mieux encore, mettre en évidence le fait qu'une illustration possible des thèses de l'une se retrouve dans les œuvres de l'autre.

35. Olga Biglieri Scurto, Barbara dei colori, op. cit., p. 315. 
Pour revenir à la peinture noétique et pour illustrer ses caractéristiques d'un point de vue théorique, il faut rappeler que plusieurs artistes élaborent en novembre 1983 un «Manifeste noétique » présenté à une exposition spectacle et à travers lequel elles se présentent publiquement à Rome au mois de février suivant, dans le lieu inattendu de Cinecittà ${ }^{36}$. Si la première ligne du manifeste affirme dans sa concision que « le noétique détermine un aspect constitutif de la pensée », Barbara donne également des éclaircissements : « la noèse, connaissance par voie intuitive, non seulement n'est pas une connaissance mineure, moins fiable, mais elle est la seule en mesure de donner une connaissance pleine, une connaissance à même de saisir le réel dans l'unité de ses différentes dimensions : matérielle, mentale, spirituelle $^{37} \gg$. Cette connaissance permet aux femmes de parler à la première personne, mais aussi de s'engager avec tout leur être, car le corps est une boussole, qui perpétuellement crée et recrée : "un corps magique, sexué $^{38} \gg$. Et la noèse, rappelant la pensée d'Irigaray, est intuition, vision enchantée, folle et magique. Le manifeste se conclut ainsi : «Instantané du moment-sans mémoire. Folie de l'instant-sans mémoire. Dimension multidirectionnelle-sans mémoire. Émotivité différente-sans mémoire. Sexualité sphérique totale sans mémoire ${ }^{39}$.

À l'occasion de la première exposition de novembre 1983, Barbara compose une œuvre novatrice, intitulée Organe de Lumière et de couleur, qu'elle décrit elle-même ainsi : "Euvre interactive à technique mixte, plus sculpture que peinture, $[\ldots]$ cinq tubes de rodoide [...], expression de peinture liquide, enveloppante, capable de transparences intrigantes ${ }^{40}$. » Quelques temps plus tard, à l'occasion d'une exposition à Bruxelles, elle enrichit son travail pour exprimer davantage son intention de prendre pour modèle le texte d'Irigaray déjà cité - le titre choisi est en effet Rencontre placentaire avec Luce -, en introduisant dans les rodoidi de petits fragments blancs sur lesquels elle écrit avec un stylo-feutre noir des phrases de la philosophe : « le jeu ne résidait pas dans la possibilité de lire ces phrases, presque toujours plongées dans la couleur liquide. Le jeu était tout symbolique : c'était une grande danse noétique entre Barbara et Luce; une danse infinie ${ }^{41} \gg$.

36. Il s'agit d'un événement multimédia car aux trois artistes signataires du manifeste s'unissent une poétesse, Fiocchetto, qui compose une poésie pour Barbara, une musicienne, Fiorella Petronici, et une danseuse, Alma Falkenberg, qui se produisent dans une performance pendant l'exposition. La critique officielle néglige l'événement à l'exception d'une recension de Claudia Salaris dans la revue Rinascita.

37. Olga Biglieri Scurto, Barbara dei colori, op. cit., p. 366.

38. Ibid., p. 361.

39. Ibid., p. 363, et Barbara dal Futurismo al 2001, op. cit., p. 99.

40. Ibid., p. 365. Organe de lumière et de couleur est une œuvre interactive, dans la mesure où les visiteurs pouvaient allumer et éteindre les couleurs, en agissant sur un clavier.

41. Ibid., p. 372. 
Combien de Barbara en Barbara? La trajectoire de l'artiste est marquée par des moments significatifs, des changements, des arrêts et des silences. Si certains critiques ont caractérisé la période placentaire-noétique comme un renvoi au credo futuriste, au refus de l'histoire et à un vitalisme projeté dans l'avenir, Barbara elle-même n'a jamais accepté ce jugement et elle s'est refusée à tracer ce type de continuité trop directe dans sa trajectoire. Elle a toutefois toujours insisté sur la rupture épistémologique produite par le féminisme, rupture dont elle a donné sa propre interprétation avec le noétique, en mettant au centre de sa trajectoire la subjectivité féminine. Il ne convient toutefois pas de séparer radicalement la richesse de la saison noétique des moments d'enrichissement antérieurs. Mis bout à bout, ils constituent en effet une sorte de manifeste, et non de testament, qui rassemble les différents traits de la figure de Barbara : si le soulèvement et le déracinement futuristes révolutionnèrent (parfois de manière douloureuse) la vie de deux générations de femmes en mouvement, ils furent nécessaires pour s'engager effectivement sur un chemin de libération permettant aux femmes d'accéder au statut de personnes et d'individualités sociales et politiques.

Francesca Brezzi

Università degli Studi Roma Tre, Dipartimento di Filosofia 